\title{
Prediction of the prognosis of somatoform disorders using the Minnesota Multiphasic Personality Inventory (MMPI)
}

Akiko Sato ( $\sim$ akikosato16@gmail.com )

Fukushima Kenritsu Ika Daigaku https://orcid.org/0000-0002-1279-4026

Syuntaro Itagaki

Fukushima Kenritsu Ika Daigaku

Takatomo Matsumoto

Fukushima Kenritu Ika Daigaku

Yoko Ise

Fukushima Kenritsu lka Daigaku

Syunya Yokokura

Fukushima Kenritsu Ika Daigaku

Tomohiro Wada

Fukushima Kenritsu Ika Daigaku

Kaoru Hayashi

Fukushima Kenritsu Ika Daigaku

Takeyasu Kakamu

Fukushima Kenritsu Ika Daigaku

Tetsuhito Fukushima

Fukushima Kenritsu Ika Daigaku

Takuya Nikaido

Fukushima Kenritsu Ika Daigaku

Shinichi Konno

Fukushima Kenritsu Ika Daigaku

Hirooki Yabe

Fukushima Kenritsu Ika Daigaku

Research article

Keywords: Hy score, consultation liaison psychiatry, Minnesota Multifaceted Personality Inventory (MMPI), Prognostic predictor, Somatoform disorders, Treatment resistant

Posted Date: March 11th, 2021 
DOI: https://doi.org/10.21203/rs.3.rs-150296/v2

License: (c) (1) This work is licensed under a Creative Commons Attribution 4.0 International License. Read Full License 
1 Prediction of the prognosis of somatoform disorders using the Minnesota

\section{Multiphasic Personality Inventory (MMPI)}

4 Akiko Sato, $\mathrm{MD}, \mathrm{PhD}^{1)}$, Shuntaro Itagaki, $\mathrm{MD}, \mathrm{PhD}^{1)}$, Takatomo Matsumto ${ }^{1)}$, Yoko Ise, $5 \quad \mathrm{MD}, \mathrm{PhD}^{1)}$, Shunya Yokokura, $\mathrm{MD}^{1)}$, Tomohiro Wada, $\mathrm{MD}^{1)}$, Kaoru Hayashi ${ }^{1)}$,

6 Takeyasu Kakamu, $\mathrm{MD}, \mathrm{PhD}^{2)}$, Tetsuhito Fukushima, $\mathrm{MD}, \mathrm{PhD}^{2)}$, Takuya Nikaido, $\mathrm{MD}$,

$7 \quad \mathrm{PhD}^{3)}$, Shinichi Konno, $\mathrm{MD}, \mathrm{PhD}^{3)}$, Hirooki Yabe, $\mathrm{MD}, \mathrm{PhD}^{1)}$

1) Department of Neuro Psychiatry, Fukushima Medical University, Hikarigaoka-1, Fukushima 960-1295, Japan

2) Department of Hygiene and Preventive Medicine, Fukushima Medical University, Hikarigaoka-1, Fukushima 960-1295, Japan

3) Department of Orthopedic Surgery, Fukushima Medical University, Hikarigaoka-1, Fukushima 960-1295, Japan

16 The study was conducted at the Department of Neuro Psychiatry, Fukushima Medical

17 University, Japan

18 Corresponding author: Akiko Sato, MD 
1 Department of Neuropsychiatry, School of Medicine Fukushima Medical University,

2 Hikarigaoka-1, Fukushima 960-1295, Japan

3 TEL: +81-24-547-1331, FAX: +81-24-548-6735

4 E-mail: akikosato16@gmail.com

$5 \quad$ All author e-mail addresses

$6 \quad$ Shuntaro Itagaki, MD, PhD; itasyun@sj8.so-net.ne.jp

$7 \quad$ Takatomo Matsumto; takarnie@icloud.com

8 Yoko Ise, MD, PhD; yokoyoko.i.2.1@gmail.com

$9 \quad$ Shunya Yokokura, MD; yukkuri916shinayo@gmail.com

10 Tomohiro Wada, MD; aokogare@gmail.com

11 Kaoru Hayashi; karl-lichter@hotmail.co.jp

12 Takeyasu Kakamu, MD, PhD; bamboo-tk@umin.ac.jp

13 Tetsuhito Fukushima, MD, PhD; t-fuku@fmu.ac.jp

14 Takuya Nikaido, MD, PhD; tnikaido@fmu.ac.jp

15 Shinichi Konno, MD, PhD; skonno@fmu.ac.jp

16 Hirooki Yabe, MD, PhD; hyabe@fmu.ac.jp

18 Department of Neuropsychiatry, School of Medicine Fukushima Medical University, 
1 Hikarigaoka-1, Fukushima 960-1295, Japan

2 TEL: +81-24-547-1331, FAX: +81-24-548-6735

3 E-mail: akikosato16@gmail.com 


\section{$1 \quad$ ABSTRACT}

2 Background: To elucidate the possibility of using the Minnesota Multifaceted

3 Personality Inventory (MMPI) to predict the prognosis of somatoform disorders, which

4 are often treatment-resistant, we investigated the prognosis of somatoform disorders

5 predicted using the MMPI.

6 Methods: During the period from January 1, 2013, to December 31, 2017, 125 cases of

7 somatoform disorder were diagnosed in the psychiatric department of Fukushima

8 Medical University Hospital, among which, 67 were consultation-liaison psychiatry

9 cases and 58 cases were only psychiatric cases. Clinical information, MMPI scores, and

10 prognosis information were collected from medical records in each case, and then

11 statistical analysis was performed.

12 Results: The results showed that the unchanged group had significantly higher scores

13 than the improved group on only the Hy scale. Receiver operating characteristic

14 analysis of the Hy scale scores of the improved and unchanged group was then

15 conducted to calculated a cutoff value. The cutoff point was 73.5 with a sensitivity of

$16 \quad 0.557$ and a specificity of 0.717 .

17 Conclusion: For patients diagnosed with somatoform disorder who had an MMPI Hy

scale score higher than the cutoff value, improvement with conventional supportive 
1 psychotherapy or drug therapy was predicted to be difficult. Therefore, the cutoff point

2 identified in this study appears to be an important index for selecting treatment for

3 somatoform disorders.

4

5 Keywords:

6 Hy score

$7 \quad$ consultation-liaison psychiatry

8 Minnesota Multifaceted Personality Inventory (MMPI)

$9 \quad$ Prognostic predictor

10 Somatoform disorders

11 Treatment-resistant

12 


\section{Background}

2 Somatoform disorders are included in the traditional clinical classification of

3 neuroses and are also classified as neurotic disorders according to the 10th revision of

4 the International Classification of Diseases (ICD-10) [1] and the Diagnostic and

5 Statistical Manual of Mental Disorders, fourth edition, text revision (DSM-IV-TR) [2,3]

6 Chronic pain is classified into nociceptive pain, neuropathic pain, and psychogenic pain

7 [4]. Among these types, psychogenic pain is classified as persistent somatoform pain

8 disorder among the somatoform disorders in the ICD-10, and as chronic pain disorder in

9 the DSM-IV-TR [5]. In addition to the distress of experiencing the symptoms

10 themselves, chronic pain is likely to cause secondary disorders such as psychiatric

11 problems and a decreased ability to carry out activities of daily living. Therefore,

chronic pain is a serious disorder that cannot be overlooked, especially economically, as

it can lead to labor loss in the productive population and increased medical expenses

because of repeated medical examinations and long-term treatment [6].

As pharmacotherapy, selective serotonin reuptake inhibitors (SSRIs), antipsychotic drugs, and benzodiazepine anxiolytics have been considerd to be useful to some extent for somatoform disorders[7]. However, although research elucidating the neural basis of somatoform disorders is currently in progress, no effective treatment has been 
1 established. Therefore, recovery from somatoform disorders is often difficult and

2 largely dependent on psychosocial treatment [8].

3 Since 1996, consultation-liaison psychiatry services, which consist of medical teams

4 including orthopedists, psychiatrists, and other co-medical staffs such as nurses,

5 physical therapists, clinical psychologists, clinical pharmacists, and social workers, have

6 been conducted in Fukushima Medical University Hospital(FMUH). These conferences

$7 \quad$ are held once a month and involve discussions on how to deal with the psychosomatic

8 problems of patients diagnosed with somatoform disorder. Owing to these conferences,

9 we have accumulated substantial MMPI data for these cases. It has been considered that

10 many patients who have psychosocial personality problems or psychiatric disorders

11 have previously consulted an orthopedist because of chronic pain and numbness or have

12 not been satisfied with conventional orthopedic treatment [5,9]. The multidisciplinary

13 nature of this conference is based on recognition that "team medical care," in which

14 related medical staffs cooperate and provide patient-centered medical care, is essential

15 to promote effective treatment and solve various problems. This liaison psychiatry

approach is characterized by a basic policy of the orthopedist remaining involved in treatment because even if the patient has psychiatric, psychological, or social problems,

18 the chief complaint is a physical symptom [5,9]. 
Numerous studies have reported personality tendencies in patients with somatoform

2 disorders based on the MMPI [10-18]. However, to our knowledge, no studies have

3 assessed the utility of the MMPI as an prognostic predictor of somatoform disorders,

4 and only a few reports have used it to predict outcomes of surgical treatment for chronic

5 back pain [19-22]. In FMUH, the MMPI has been continuously conducted, and data

6 have been accumulated on the cases discussed in the liaison conferences for the purpose

7 of evaluating whether patients with chronic pain suffer from latent paranoia, depression,

8 or other psychiatric disorders, as well as whether their personality may affect their

9 symptoms $[23,24]$.

10 Although the MMPI has mainly been used for diagnosis and assessment, if it could

11 be used for the prediction of prognosis of somatoform disorders, treatment would be

expected to proceed more smoothly because more effective interventions could be started at an early stage, and the patient could recognize the therapeutic effects sooner.

14 In addition, considering that MMPI takes quite much time to be completed due to a

15 large number of question items, over 500, if key items predicting negative outcome could be identified, it should be more useful and reduce psychological burden of target patients. 
1 factors associating with negative outcome of somatoform disorders, another is to

2 identify key items of MMPI predicting negative outcome. Therefore, we collected the

3 data from patients who all had received MMPI in clinical settings, classified them into

4 two groups (improvedgroup vs. unchanged group) based on the chart review, and

5 examined two groups.

$6 \quad$ Methods

$7 \quad$ ParticipantsDuring the period from January 1, 2013, to December 31, 2017, 125

8 cases of somatoform disorder were diagnosed at the psychiatric department of

9 Fukushima Medical University Hospital based on the ICD-10 [1]. Among these cases,

1067 were associated with the consultation-liaison psychiatry approach, and 58 with only

11 the psychiatric approach. Also among these cases, 80 were classified as Persistent

12 somatoform pain disorder, 31 were as Somatization disorder, 8 were as Undifferentiated

13 somatoform disorder, 4 were as Somatoform autonomic dysfunction, and 2 were as

14 Other somatoform disorders. All these cases were treated conventional supportive

15 psychotherapy or pharmacotherapy.

Measurements and procedures

18 The Minnesota Multifaceted Personality Inventory (MMPI) is a standardized 
1 psychometric test of adult personality and psychopathology based on the questionnaire

2 method developed by Hathaway and McKinley of the University of Minnesota in the

3 late 1930s [25,26]. The MMPI is composed of 550 items, and hundreds of additional

4 scales have been developed. In the United States, a re-standardization of the MMPI

5 began around the end of the 1980s because of problems with the wording of the item

6 text and inadequate standardization procedures in the original version. The second

7 version, the MMPI-2, maintained continuity with the original. The Japanese version of

8 the MMPI was published in 1963, but mistranslations and problems with

9 standardization procedures were apparent from the beginning, and efforts to resolve

10 these problems began around 1990. The New Japan Version of the MMPI was published

11 in 1993, and is still currently used in Japan [25,26]. The original purpose of the MMPI

was to provide objective information necessary for psychiatric diagnosis. Subsequently, the purpose shifted to personality assessment, and thus, it is now one of the most

14 frequently used personality tests around the world [25,26]. Actually, more than 12,000 papers have been published on the MMPI and MMPI-2 since the late 1940s [27]. information on factors that may affect the prognosis of somatoform disorders for each case, including age, gender, duration of illness, the comorbidity of developmental 
1 disorders, decreased cerebral blood flow, history of surgery, MMPI profile, and presence

2 of the conversion V pattern on the MMPI. Then, we identified patients indicating

3 negative outcome based on the following information obtained from the charts : (1)

4 Subjective estimation regarding pain, (2) Social function including ADL. This group

5 was named "improved group (IG)" and others named "non-improved group (NIG)".

6 We profiled four validity scales (?, L, F, K; Table 1) and 10 clinical scales (Hs, D,

7 Hy, Pd, Mf, Pa, Pt, Sc, Ma, Si; Table 2) as basic scales for the MMPI [9,28]. The

8 interpretation of the conversion $\mathrm{V}$ pattern is shown in Table 3. Decreased cerebral blood

9 flow was defined as when a radiologist reported that "there was low blood flow(Vd less

10 than $30 \mathrm{ml} / \mathrm{ml}$ by ARG method)" based on $\mathrm{N}$-isopropyl-( $\left.{ }^{123} \mathrm{I}\right) p$-iodoamphetamine

11 computed tomography, regardless of the brain region. The comorbidity of

12 developmental disorders was defined as when a psychiatrist noted autism spectrum

13 disorder (ASD), attention deficit hyperactivity disorder (ADHD), or pervasive

14 developmental disorder in a patient's medical records. A history of surgery was defined

15 as any descriptions of orthopedic surgery in a patient's medical records.

$17 \quad$ Statistical analysis

18 We descriptively compared each factor between the IG and NIG groups. Differences 
1 between groups were analyzed using the Mann-Whitney U test, the Student $t$-test, and

2 the chi-squared test. Among the MMPI scales, receiver operating characteristic (ROC)

3 curves that showed a significant difference between the IG and NIG were created for the

4 Hy scale. The area under the curve (AUC) and 95\% confidence intervals (CIs) were

5 calculated, as was the cutoff value using Youden's index. Statistical analysis was

6 performed using SPSS ver. 26 (SPSS, Chicago, IL, USA), and $p$ values $<0.05$ were

7 considered statistically significant.

\section{$9 \quad$ Results}

10 In total, 125 patients (49 males, 76 females; mean age \pm standard deviation [SD],

$1151.9 \pm 17.4$ years) participated in this study.

Prognosticcomparison of the participants'basic characteristics (Table 4)

14 No significant differences in age, gender, duration of illness, the comorbidity of developmental disorders, decreased cerebral blood flow, or history of surgery were observed between the IGand NIG. The mean age \pm SD of the IG and NIG were $49.3 \pm$ 17.8 and $53.8 \pm 17.5$, respectively $(p=0.167)$. Regarding the gender ratio, $16(34.6 \%)$ 
$146(58.2 \%)$ females were in the unchanged group $(p=0.44)$. The median duration of

2 illness in the IG was 35 months (25th-75th percentile $=24-91$ ), and that in the NIG was

354 months $(25$ th -75 th percentile $=24-120 ; p=0.168)$. The numbers of patients with

4 decreased cerebral blood flow were $14(51.9 \%)$ in the IG and $30(57.7 \%)$ in the NIG ( $p$

$5=0.62)$. The numbers of patients who had a history of surgery were $15(32.6 \%)$ in the

$6 \quad$ IG and $26(32.9 \%)$ in the NIG $(p=0.972)$.

$7 \quad$ No significant differences were found in the presence of the conversion $\mathrm{V}$ pattern

8 between the IG and NIG (17.4\% vs. $17.7 \%$, respectively; $p=0.963)$. Regarding the

9 presence of developmental disorders, the comorbidity rate of developmental disorders

10 was $10.9 \%$ in the IG and $25.3 \%$ in the NIG; this rate tended to be higher in the

11 unchanged than in the improved group, but this difference was not significant ( $p=$

$12 \quad 0.051)$

Prognostic comparison of each MMPI scale (Table 5) group showed a significantly higher value than the IG on the Hy scale (IG, $66.2 \pm 15.4$ vs. NIG, $73.5 \pm 12.4 ; p=0.04)$. The scores on the ? scale were $45.4 \pm 11.1$ for the IG and $49.0 \pm 9.3$ for the NIG; although the IG tended to have lower scores, this difference 
1 was not significant $(p=0.051)$.

2

\section{ROC curves of the Hy score for all participants (Figure 1)}

4

The results of the ROC analysis performed using the Hy scores of all participants

5 indicated a significant difference between the IG and NIG groups, with an AUC (95\%

6 CI) of $0.652(0.55-0.753)$. The cutoff point was 73.5 with a sensitivity of 0.557 and a

$7 \quad$ specificity of 0.717 .

\section{Discussion}

The MMPI can identify the personality of subjects from multiple aspects based on answers to questions assessing, for example, hypochondriac, obsessive, and compulsive tendencies. A configuration in which the Hypochondriasis (Hs) and Hysteria (Hy) scale are $\mathrm{T}=70$ or more and the $\mathrm{T}$ score of these two scales is 10 or higher than that of the

14 Depression (D) scale is called the "conversion V" pattern. The conversion V pattern suggests that subjects tend to "replace" their psychological problems with socially acceptable ones, such as physical complaints. Tendencies to escape from a situation through physical complaints, to try and control others, and to suppress or deny the problem are then presumed $[9,10]$. 
1 Then, We carefully discuss each result in detail as follows.

2

\section{Comparison between the improved and unimproved prognosis groups}

We selected basic characteristics such as age, gender, duration of illness, the comorbidity of developmental disorders, decreased cerebral blood flow, history of surgery, and the conversion V pattern on the MMPI as factors that may affect the outcomeof somatoform disorders. An analysis of each outcome group did not reveal any

significant differences. Regarding the cerebral blood flow, it has been reported to be decreased in patients with chronic pain[29]. But no significant differences were found in this study. But the NIG was more likely to have developmental disorders. It has been reported that among developmental disorders in children, both ASD and ADHD are associated with a high rate of chronic pain [30,31]. For ASD and ADHD, it is said that a therapeutic effect can be obtained by combining psychosocial treatment in addition to pharmacotherapy [32,33]. Therefore, when the comorbidity of developmental disorders is recognized, it is thought that somatoform disorders could be improved by performing a therapeutic intervention particularly for developmental disorders. In addition, analysis of each scale of the MMPI showed that only the Hy scale had a significant difference, indicating that the NIG had higher scores on the Hy scale than the improved 
1 group. We discuss about what this result mean in detail as follows.

2

3

4

5

6

7

\section{Significance of high scores on the Hy scale}

A high score on the Hy scale indicates a tendency to avoid responsibilities related to psychological conflicts by converting these to physical symptoms (a tendency to use conversion symptoms). It also means that individuals with a high Hy score tend to be immature and lack self-insight, indicating that their relationships with other people are often superficial, even though they may appear to be appear to be socially well adopted [9]. A significant difference was observed between the IGand NIG only in this Hy scale score. Therefore, ROC analysis was performed on the Hy score for the IG and NIG, resulting in a cutoff score of 73.5. Previous studies have reported that patients with chronic pain show higher Hy scores $[34,35]$. On the other hand, when scores on the K and Hs scales are low, only a high Hy scale score is considered insufficient to consider whether the pain is psychogenic [36,37].

However, even if only a high Hy scale score is insufficient to determine whether the pain is psychogenic, a high Hy score is still considered to indicate a remarkably severe degree of distress in terms of physical symptoms. In addition, all cases analyzed in this study had already been diagnosed as somatoform disorder. Few reports have been published on the outcome viewpoint of the MMPI for somatoform disorders, and no 
1 reported cases have shown meaningful profiles or characteristics for each scale [38].

2 The cutoff point calculated in this study was 73.5, which was even higher than the

3 score generally considered to be abnormal (70). If the Hy scale score of a patient

4 diagnosed with a somatoform disorder is higher than this cutoff value, he or she is

5 considered difficult to treat with conventional supportive psychotherapy or

6 pharmacotherapy. In such cases, it may be necessary to consider psychiatric

7 "multidisciplinary" treatment, which is a further enhancement of conventional

8 treatments [39-43], e.g., psychosocial treatment such as cognitive behavioral therapy

9 [44-48] or mindfulness therapy [49], pharmacotherapy, and environmental adjustments.

10 Therefore, this cutoff point appears to be an important index for treatment selection in

11 patients with somatoform disorder. However, since this was a retrospective study, if the

12 Hy scale score exceeds the cutoff point, prospective studies are needed to compare the

13 prognoses of the following two groups: one that is provided with therapeutic

14 interventions such as augmented pharmacotherapy and psychotherapy, psychosocial

15 treatment, and environmental adjustments, and another that receives standard therapy

16 (general pharmacotherapy and supportive psychotherapy).

\section{Limitations}


1 The classification of the outcome of somatoform disorder among the current group

2 used in this study was based on only the chart review not more reliable ways such as

3 diagnostic (structured) interviews or self-administered questionnaires.

7 predicting the prognosis of patients with somatoform disorders based on the MMPI. The

8 results suggested that the Hy scale score might influence the prognosis. The cases

9 exceeding the cutoff point based on ROC analysis are considered difficult to treat with conventional supportive psychotherapy or pharmacotherapy. Therefore, this cutoff point

11 could be an important index in considering treatment options for improving the prognosis of patients with somatoform disorders.

\section{Declarations}

Ethics approval and consent to participate : This study was approved by the Ethics

16 Committee of Fukushima Medical University (approval No. 2941).

18 Consent for publication : Consent to publish this study was provided, and all 
1 participants signed an informed consent form.

2

3 Availability of data and material : Detailed data are available from the corresponding

4 authors upon reasonable request.

5

6 Competing interests : The authors have no competing interests to report.

7

8 Funding : This research did not receive any specific grant from funding agencies in the

9 public, commercial, or not-for-profit sectors.

11 Authors' contributions : AS conceived the study.AS and KH collected the data.AS

and TK analyzed the data. All authors contributed to data analysis, drafting and

critically revising the paper, gave final approval of the version to be published, and

agreed to be accountable for all aspects of the work.

16 Acknowledgements : We sincerely thank all of the orthopedic surgeons, psychiatrists,

17 and medical staffs involved in liaison therapy at Fukushima Medical University. 
1 Authors' information : Department of Neuro Psychiatry, Fukushima Medical

2 University, Hikarigaoka-1, Fukushima 960-1295, Japan

3 Statement : The pre-print version of this article is present and can be accessed on

4 https://www.researchsquare.com/article/rs-150296/v1

5 This article is not published nor is under publication elsewhere

6

7 


\section{$1 \quad$ References}

2 [1] World Health Organization: ICD-10, the ICD-10 Classification of Mental and

3 Behavioural Disorders: Clinical Descriptions and Diagnostic Guidelines, World Health

4 Organization; 1992.

5 [2] American Psychiatric Association; Diagnostic and Statistical Manual of Mental

6 Disorders: DSM IV, American Psychiatric Association; 1994.

7 [3] T. Okuma; Modern Clinical Psychiatry, 12th ed.:Kaneharashuppan; 2013.

8 [4] K. Watanabe, S. Konno. Musculoskeletal pain management. Prog Med;2013;33 :9-

912.

10 [5] H. MashikoIndefinite complaints in orthopedic surgery. Japanese Journal of Clinical

11 Psychiatry:2012; 41: 275-281.

12 [6] Y. Mizuno, M. Fukunaga, Y. Nakai.Differences in psychological characteristics

13 between chronic pain patients and other psychosomatic (mind-body) disease patient.

14 The Journal of the Japanese Society for the Study of Chronic Pain:2004; 23: 193-199.

15 [7] Y. Nagoshi. Recent advances in pharmacotherapy for somatic symptom and related

16 disorders (somatoform disorders). Japanese Journal of Psychosomatic Medicine.2019;

$17 \quad 59 ; 554-559$.

18 [8] M. Sellbom, D. Wygant, M. Bagby. Utility of the MMPI-2-RF in detecting non- 
1 credible somatic complaints, Psychiatry Res.2012; 197;295-301.

2 [9] K. Otani. Liaison treatment for patients with chronic pain of locomotive organ-

3 experience of Fukushima Medical University Hospital, Japanese Journal of

$4 \quad$ Psychosomatic Medicine.2011; 51;709-714.

$5 \quad$ [10] B. Naylor, S. Boag, S.M. Gustin. New evidence for a pain personality? A critical

6 review of the last 120 years of pain and personality, Scand. J. Pain.2017; 17; 58-67.

7 [11] A.A. Vendrig. The Minnesota Multiphasic Personality Inventory and chronic pain,

8 a conceptual analysis of a long-standing but complicated relationship. Clin. Psychol.

$9 \quad 2000 ;$ Rev. 20; 533-559.

10 [12] D.A. Fishbain, B. Cole, R.B. Cutler, J. Lewis, H.L. Rosomoff, R.S.

11 Rosomoff.Chronic pain and the measurement of personality: do states influence traits?,.Pain Med.2006; 7;509-529.

[13] J. Dersh, P.B. Polatin, R.J. Gatchel.Chronic pain and psychopathology, research findings and theoretical considerations, Psychosom. 2002;Med. 64;773-786.

Donias, et al.. Somatoform disorders: comorbidity with other DSM-III-R psychiatric diagnoses in Greece, Compr. Psychiatry.1999; 40;299-307. 
1 al..Examination of the Modified Somatic Perception Questionnaire (MSPQ) in a large

2 sample of credible and noncredible patients referred for neuropsychological testing,

3 Clin. Neuropsychol. 2018;32; 165-182

4 [16] J.A. Koelen, E.H.M. Eurelings-Bontekoe, S.A.M. van Broeckhuysen-Kloth, W.M.

5 Snellen, P. Luyten. Social cognition and levels of personality organization in patients

6 with somatoform disorders, a case-control study.2014; J. Nerv. Ment. Dis. 202;217-223

7 [17] A.A. Vendrig, J.J. Derksen, H.R. de Mey. MMPI-2 Personality Psychopathology

8 Five (PSY-5) and prediction of treatment outcome for patients with chronic back pain.

9 2000;J. Pers. Assess;74;423-438.

10 [18] M. Hasegawa, S. Hattori, M. Ohnaka, K. Ishizaki, F. Goto.Psychological

11 characteristics of chronic pain patients. 1998;Journal of Japan Society of Pain Clinicians

$125 ; 30-35$.

13 [19] D. Barnes, R.J. Gatchel, T.G. Mayer, J. Barnett. Changes in MMPI profile levels of

14 chronic low back pain patients following successful treatment.1990; J. Spinal Disord. 3;

$15 \quad 353-355$.

16 [20] C. McCreary, B. Naliboff, M. Cohen.A comparison of clinically and empirically

17 derived MMPI groupings in low back pain patients.1989; J. Clin. Psychol. 45;560-570.

18 [21] D.E. Williams, J.K. Thompson, J.D. Haber, J.M. Raczynski. MMPI and headache: 
1 a special focus on differential diagnosis, prediction of treatment outcome, and patient-

2 treatment matching.1986; Pain 24; 143-158.

3 [22] C. McCreary, J. Turner, E. Dawson.The MMPI as a predictor of response to

4 conservative treatment for low back pain.1979;J. Clin. Psychol. 35;278-284.

5 [23] S. Kasahara. Psychiatric approach for the patients with chronic pain, Japanese

6 Journal of Clinical Psychiatry.2013;42;739-748.

7 [24] T. Nikaido, S. Yabuki, K. Otani, K. Watanabe, K. Kato, Y. Kobayashi, et

8 al..Scientific approach for pain based on biopsychosocial model: liaison approach for

9 chronic low back pain.2016; Journal of Musculoskeletal Pain Research 8; 192-198.[25]

10 T. Shioya.History of MMPI, The Society for MMPI New Japanese Version MMPI.2004.

11 http://www.mmpi-new.com/history.Accessed 18 April 2020. [26] H. Noro, W. Arakawa,

12 S. Ide. MMPI Handbook, Basics to Understand, Japanese Clinical Society for the Study

13 of MMPI.Tokyo: Kongoshuppan; 2014.

14 [27] J.N. Butcher. MMPI-2 in Psychological Treatment. Oxford: Oxford University

15 Press; 1990.

16 [28] Kanazawa University Psychology Laboratory.MMPI Japanese Language

17 Implementation Guide (Kanazawa University version). Kanazawa: Kanazawa

18 University Psychology Laboratory; 1965. 
1 [29] Shin-ichi Konno, Miho Sekiguchi. Association between brain and low back

$2 \quad$ pain.2018;J Orthop Sci 23; 3-7.

3 [30] C.W. Lipsker, S. Bölte, T. Hirvikoski, M. Lekander, L. Holmström, R.K. Wicksell.

4 Prevalence of autism traits and attention-deficit hyperactivity disorder symptoms in a

5 clinical sample of children and adolescents with chronic pain.2018; J. Pain Res. 8;

$6 \quad 2827-2836$.

7 [31] C. Baeza-Velasco, D. Cohen, C. Hamonet, E. Vlamynck, L. Diaz, C. Cravero, et

8 al..Autism, joint hypermobility-related disorders and pain.2018; Front. Psychiatry 9 ;

9656.

10 [32] J.J.S. Kooij, D. Bijlenga, L. Salerno, R. Jaeschke, I. Bitter, J. Balázs, et al..

11 Updated European Consensus Statement on diagnosis and treatment of adult

12 ADHD.2019; Eur. Psychiatry 56;14-34.

13 [33] L. Bishop-Fitzpatrick, N.J. Minshew, S.M. Eack.A systematic review of

psychosocial interventions for adults with autism spectrum disorders. 2013;J. Autism

Dev. Disord. 43;687-694.

[34] S.R. Ornduff, A.F. Brennan, C.L. Barrett.The Minnesota Multiphasic Personality 
1 [35] B.N. Kinder, G. Curtiss, S. Kalichman. Anxiety and anger as predictors of MMPI

2 elevations in chronic pain patients.1986; J. Pers. Assess. 50;651-661.

3 [36] M. Aragona, L. Tarsitani, S. De Nitto, M. Inghilleri. DSM-IV-TR “pain disorder

4 associated with psychological factors" as a nonhysterical form of somatization.2008;

$5 \quad$ Pain Res. Manag.;13;13-18.

6 [37] R.E. McGrath, W.B. O’Malley. The assessment of denial and physical complaints:

7 the validity of the Hy scale and associated MMPI signs.1986; J. Clin.

$8 \quad$ Psychol. ; 42;754-760.

9 [38] A.M. Tarescavage, J. Scheman, Y.S. Ben-Porath. Prospective comparison of the

10 Minnesota Multiphasic Personality Inventory-2 (MMPI-2) and MMPI-2-Restructured

11 Form (MMPI-2-RF) in predicting treatment outcomes among patients with chronic low

12 back pain.2018; J. Clin. Psychol. Med. Settings 25;66-79.

13 [39] J.A. Koelen, J.H. Houtveen, A. Abbass, P. Luyten, E.H. Eurelings-Bontekoe, S.A.

14 Van Broeckhuysen-Kloth, et al.. Effectiveness of psychotherapy for severe somatoform

15 disorder: meta-analysis.2014; Br. J. Psychiatry 204;12-19.

16 [40] A.M. Sutherland, J. Nicholls, J. Bao, H. Clarke. Overlaps in pharmacology for the

17 treatment of chronic pain and mental health disorders.2018; Prog.

18 Neuropsychopharmacol. Biol. Psychiatry;87; 290-297. 
1 [41] N. van Dessel, M. den Boeft, J.C. van der Wouden, M. Kleinstäuber, S.S. Leone,

2 B. Terluin, et al..Non-pharmacological interventions for somatoform disorders and

3 medically unexplained physical symptoms (MUPS) in adults.2014; Cochrane Database

4 Syst. Rev. ;CD011142,.https://doi.org/10.1002/14651858.CD011142.pub2. Accessed 18

5 April 2020.

6 [42] H. van Ravesteijn. [Mindfulness-based cognitive therapy for patients with

7 somatoform disorders].2016; Tijdschr. Psychiatr; 58;198-206.

8 [43] K. Cyranka, K. Rutkowski, M. Mielimąka, J.A. Sobański, B. Smiatek-Mazgaj, K.

9 Klasa, et al.. Changes in personality functioning as a result of group psychotherapy with

10 elements of individual psychotherapy in persons with neurotic and personality disorders

11 - MMPI-2.2016; Psychiatr. Pol.; 50;105-126.

12 [44] S.L. Kurlansik, M.S. Maffei. Somatic Symptom Disorder.2016; Am. Fam.

13 Physician;93; 49-54.

14 [45] A. Yoshino, Y. Okamoto, R. Jinnin, K. Takagaki, A. Mori, S. Yamawaki.Role of

15 coping with negative emotions in cognitive behavioral therapy for persistent

16 somatoform pain disorder: is it more important than pain

17 catastrophizing?.2019;Psychiatry Clin. Neurosci. ;73;560-565.

18 [46] A. Yoshino, Y. Okamoto, M. Doi, M. Horikoshi, K. Oshita, R. Nakamura, et al.. 
1 Effectiveness of group cognitive behavioral therapy for somatoform pain disorder

2 patients in Japan: a preliminary non-case-control study,;2015;Psychiatry Clin.

$3 \quad$ Neurosci. ;69;763-772.

4 [47] J. Liu, N.S. Gill, A. Teodorczuk, Z.J. Li, J. Sun.The efficacy of cognitive

5 behavioural therapy in somatoform disorders and medically unexplained physical

6 symptoms: a meta-analysis of randomized controlled trials.2019;J. Affect. Disord.;245 ;

$7 \quad 98-112$.

8 [48] A. Sumathipala.What is the evidence for the efficacy of treatments for somatoform

9 disorders? A critical review of previous intervention studies.2007;Psychosom.

10 Med. ;69;889-900.

11 [49] L. Hilton, S. Hempel, B.A. Ewing, E. Apaydin, L. Xenakis, S. Newberry, et al..

12 Mindfulness meditation for chronic pain: systematic review and meta-analysis.2017;

13 Ann. Behav. Med.;51;199-213.

17 Figure legend

19 Fig. 1. Receiver operating characteristic (ROC) curves of the Hysteria (Hy) scale score 
1 on the Minnesota Multiphasic Personality Inventory (MMPI) for all participants. ROC

2 analysis was performed using the Hy scale scores for all participants that showed a

3 significant difference between the improved and unchanged groups; the area under the

4 curve (AUC) (95\% confidence interval) was $0.652(0.55-0.753)$, as calculated using

$5 \quad$ Youden's index, with a cutoff value of 73.5.

6

7 
Figures

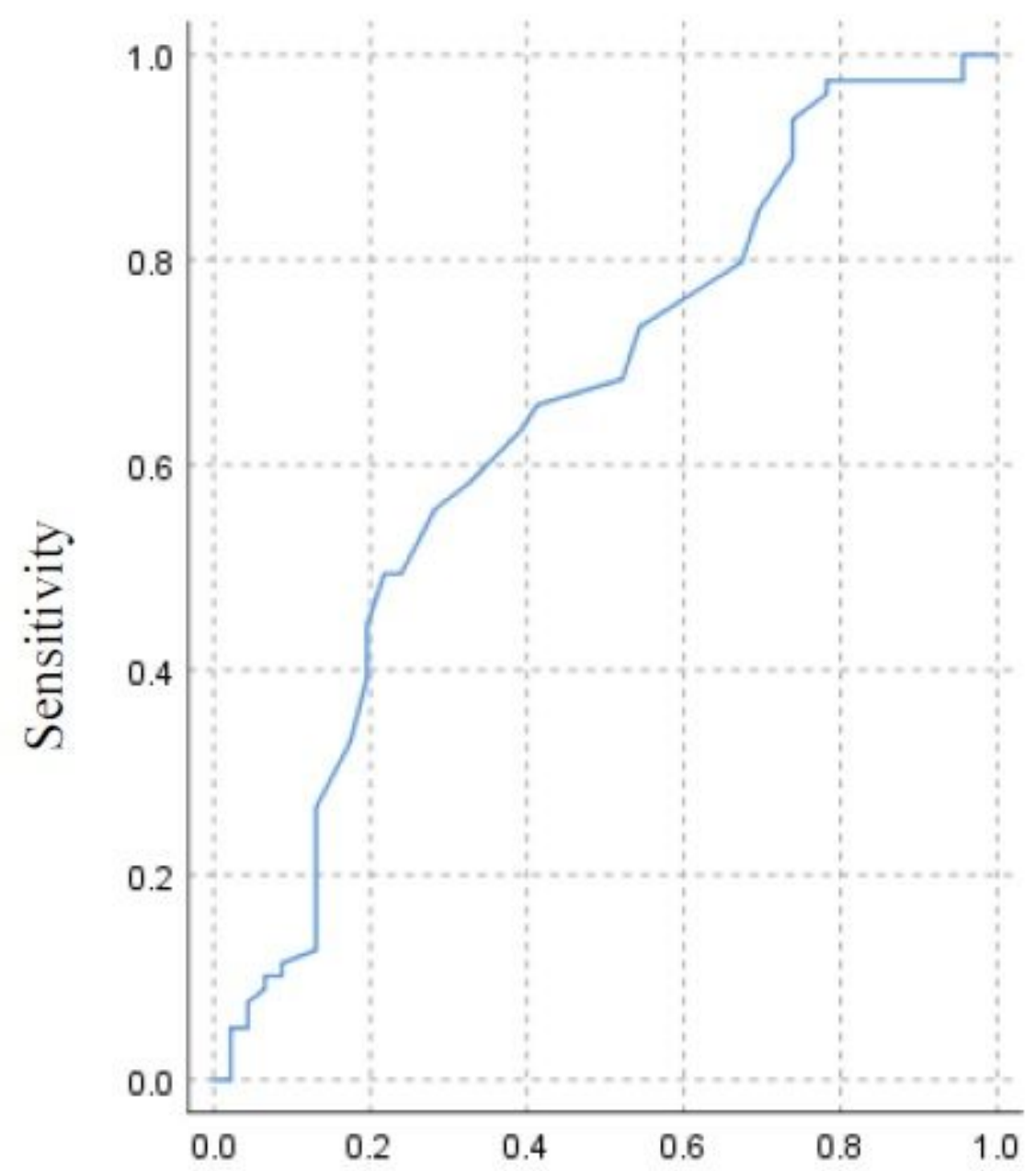

1-Specificity

\begin{tabular}{|c|c|c|}
\hline $\begin{array}{c}\text { AUC } \\
(95 \% \text { confidence interval })\end{array}$ & $p$ & Cutoff value \\
\hline $0.652(0.55-0.753)$ & 0.004 & 73.5 \\
\hline
\end{tabular}

Figure 1

ROC curves of the Hy score for all participants

Supplementary Files 
This is a list of supplementary files associated with this preprint. Click to download.

- Tables.pdf 